Revised for publication 1/13/99

\title{
POINT DEFECT CLUSTER FORMATION IN IRON DISPLACEMENT CASCADES UP TO $50 \mathrm{keV}$
}

\author{
ROGER E. STOLLER \\ Metals and Ceramics Division \\ Oak Ridge National Laboratory \\ P.O. Box 2008, Oak Ridge, TN 37831-6376 \\ Presented at the MRS 1998 Fall Meeting \\ SYMPOSIUM N: Microstructural Processes in Irradiated Materials \\ November 30 to December 4, 1998 \\ Boston, MA
}

Keywords: computer simulations, displacement cascades, molecular dynamics, point defects, radiation damage

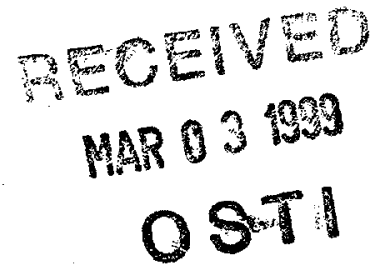

"The submitted manuscript has been authored by a contractor of the U.S. Government under contract No.

DE-AC05-960R22464. Accordingly,

the U.S. Government retains a

nonexclusive, royalty-free license

to publish or reproduce the published

form of this contribution, or allow others

to do so, for U.S. Government purposes." 


\section{ROGER E. STOLLER}

Metals and Ceramics Division, Oak Ridge National Laboratory, P.O. Box 2008, Oak Ridge, TN 37831-6376, USA, rkn@ornl.gov

\section{ABSTRACT}

The results of molecular dynamics displacement cascade simulations in iron at energies up to $50 \mathrm{keV}$ and temperatures of 100,600 , and $900 \mathrm{~K}$ are summarized, with a focus on the characterization of interstitial and vacancy clusters that are formed directly within the cascade. The fraction of the surviving point defects contained in clusters, and the size distributions of these incascade clusters have been determined. Although the formation of true vacancy clusters appears to be inhibited in iron, a significant degree of vacancy site correlation was observed. These well correlated arrangements of vacancies can be considered nascent clusters, and they have been observed to coalesce during longer term Monte Carlo simulations which permit short range vacancy diffusion. Extensive interstitial clustering was observed. The temperature and cascade energy dependence of the cluster size distributions are discussed in terms of their relevance to microstructural evolution and mechanical property changes in irradiated iron-based alloys.

\section{INTRODUCTION}

Primary damage formation in irradiated materials has been extensively investigated using the method of molecular dynamics (MD). ${ }^{1-9}$ Modern, high speed computers with large amounts of memory have recently enabled researchers to examine higher energy events with their requirement for larger numbers of atoms. These same computing resources have permitted cascade simulations to be completed in sufficient numbers to allow for statistically meaningful trends to be determined as a function of simulation energy and irradiation temperature, and for comparisons to be made between different materials. For example, the comparison of iron and copper contained in Ref. 5 is based on a database of over 600 cascades. Cascade energies from $60 \mathrm{eV}$ to $10 \mathrm{keV}$ were used at irradiation temperatures of 100,600 , and $900 \mathrm{~K}$.

The results presented here extend those of the collaborative study presented in Ref. 5, with the peak cascade energy reaching $50 \mathrm{keV}$ at $100 \mathrm{~K}$ and $20 \mathrm{keV}$ at $600 \mathrm{~K}$. The higher energy simulations provide an opportunity to validate the trends established at lower energies. In particular, they probe the domain of extensive subcascade formation. Both the total number of point defects produced and the fraction of these defects that are found in clusters at the end of the cascade event appeared to be relatively independent of temperature at the lower energies. The incascade cluster size distributions exhibit more temperature dependence at the higher energies, with vacancy clustering decreasing and interstitial clustering increasing as the temperature increases.

\section{SIMULATION METHOD}

The calculations were carried out using the MOLDY ${ }^{10} \mathrm{MD}$ code and the interatomic potential for iron developed by Finnis and Sinclair for $\alpha$-iron ${ }^{11}$ and subsequently modified by Calder and Bacon. ${ }^{3}$ This code and potential have been widely used, and are discussed in detail elsewhere. ${ }^{2-9}$ The simulations were carried out using periodic boundary conditions and at constant pressure. Since the boundary atoms were not damped to remove heat, the initiation of a cascade event led to some lattice heating. The primary impact of the temperature increase is to extend the lifetime of the thermal spike, it has no impact on the ballistic phase of the cascade. Furthermore, the temperature increase does not appear to influence defect survival. ${ }^{3,5,9}$ However, 


\section{DISCLAIMER}

This report was prepared as an account of work sponsored by an agency of the United States Government. Neither the United States Government nor any agency thereof, nor any of their employees, make any warranty, express or implied, or assumes any legal liability or responsibility for the accuracy, completeness, or usefulness of any information, apparatus, product, or process disclosed, or represents that its use would not infringe privately owned rights. Reference herein to any specific commercial product, process, or service by trade name, trademark, manufacturer, or otherwise does not necessarily constitute or imply its endorsement, recommendation, or favoring by the United States Government or any agency thereof. The views and opinions of authors expressed herein do not necessarily state or reflect those of the United States Government or any agency thereof. 


\section{DISCLAIMER}

Portions of this document may be illegible in electronic image products. Images are produced from the best available original document. 
there is some evidence that the adiabatic conditions may have an impact on the incascade interstitial clustering fraction. ${ }^{12}$

Prior to conducting the cascade simulations, a block of atoms was thermally equilibrated for about 10 ps. This equilibrated atom block was used as the starting point for the subsequent cascade simulations; the size of the block increased for higher energy simulations as shown in Table 1. The simulations were initiated by giving a lattice atom the specified amount of kinetic energy and an initial direction. Multiple cascades were differentiated by changing the location of the primary knock-on atom (PKA) or by using a different equilibrated atom block. As discussed in Ref. 5, the initial kinetic energy is analogous to the damage energy in the standard NRT displacement model. ${ }^{13}$ The difference between these two values is the kinetic energy lost to electronic excitation; this energy loss mechanism is not simulated in the MOLDY code. ${ }^{5}$ In addition to the MD simulation energies, Table 1 lists the corresponding PKA energies. The neutron energies listed in Table 1 are those for which the PKA energy is the average recoil energy in an elastic collision. Note that these MD simulations account for recoils from neutrons that are well into the high (fast) energy domain.

Table 1: Typical MD cascade parameters and required atom block sizes

\begin{tabular}{|c|c|c|c|c|}
\hline $\begin{array}{c}\text { Neutron } \\
\text { Energy }(\mathrm{MeV})\end{array}$ & $\begin{array}{c}\text { Average PKA } \\
\text { Energy (keV) }\end{array}$ & $\begin{array}{c}\text { Corresponding } \\
\mathrm{E}_{\mathrm{MD}}(\mathrm{keV})\end{array}$ & $\begin{array}{c}\text { NRT } \\
\text { Displacements }\end{array}$ & $\begin{array}{c}\text { Atoms in } \\
\text { Simulation }\end{array}$ \\
\hline 0.0034 & 0.116 & $\mathbf{0 . 1}$ & 1 & 3,456 \\
\hline 0.0058 & 0.236 & $\mathbf{0 . 2}$ & 2 & 6,750 \\
\hline 0.014 & 0.605 & $\mathbf{0 . 5}$ & 5 & 6,750 \\
\hline 0.036 & 1.24 & $\mathbf{1}$ & 10 & 54,000 \\
\hline 0.074 & 2.54 & $\mathbf{2}$ & 20 & 54,000 \\
\hline 0.19 & 6.60 & $\mathbf{5}$ & 50 & 128,000 \\
\hline 0.40 & 13.7 & $\mathbf{1 0}$ & 100 & 250,000 \\
\hline 0.83 & 28.8 & $\mathbf{2 0}$ & 200 & 250,000 \\
\hline 2.3 & 78.7 & $\mathbf{5 0}$ & 500 & $2,249,728$ \\
\hline
\end{tabular}

\section{INCASCADE CLUSTERING IN MD SIMULATIONS}

\section{Interstitial Clustering}

The primary parameters that have been used to describe the results of cascade simulations are the total number of surviving point defects and the fraction of the surviving defects that are contained in clusters. ${ }^{2-10,14,15}$ The surviving defects are often expressed as a fraction of the NRT displacements as listed in Table $1,{ }^{13}$ whereas the number in clusters has been expressed as either a fraction of the NRT displacements or a fraction of the total surviving MD defects. In this study, the criterion used in defining clusters of point defects was that each defect in the cluster must be in a nearest neighbor position to another defect. Under this definition, defect clusters are not necessarily compact structures.

The dependence of incascade interstitial clustering on cascade energy is shown in Fig. 1 for simulation temperatures of 100,600 , and $900 \mathrm{~K}$, where the number of interstitials in clusters has been divided by the corresponding number of NRT displacements. At each energy, the data point is an average of from 7 to 24 cascades and the standard deviations about the average are shown at the highest energies. The solid line through the points is a least-squares fit to the energy dependence that was used in an assessment of neutron energy spectrum effects. ${ }^{14}$ The points at 10 and $20 \mathrm{keV}$ have been slightly offset along the energy axis to help distinguish between the results. 


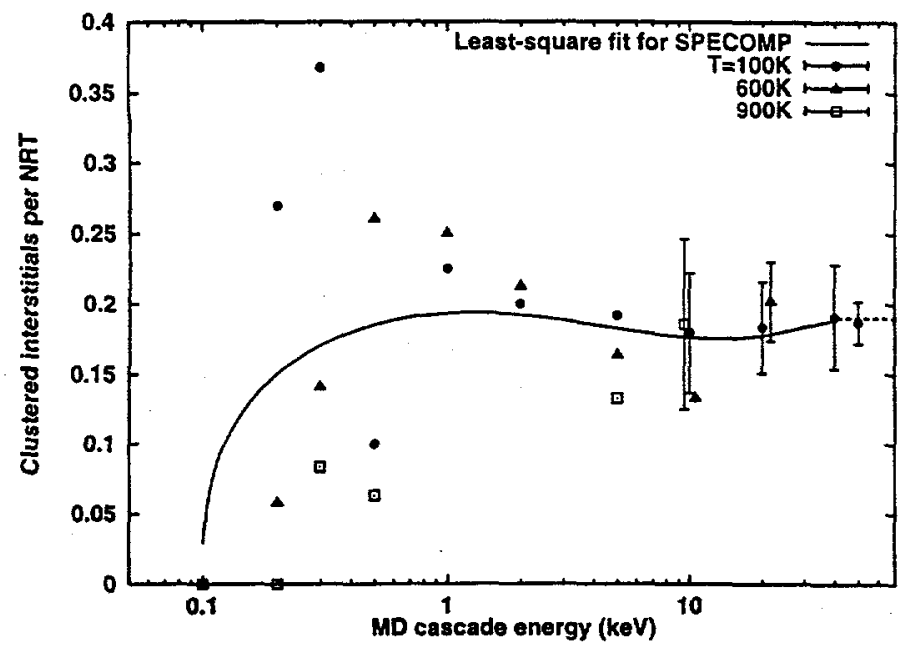

Fig. 1 Incascade interstitial clustering fraction: clustered interstitials divided by NRT displacements.

Considerable scatter is observed in the clustering fraction at low energies, and there appears to be only a weak dependence on temperature. Overall, the interstitial clustering fraction exhibits more variability between cascades at the same energy than does defect survival. The standard deviations in Fig. 1 are much larger than the corresponding values for the defect survival fraction. ${ }^{14,15}$ The fraction of interstitials in clusters appears to stabilize at about 18 to $20 \%$ of the NRT displacements above $5 \mathrm{keV}$, which corresponds to about $60 \%$ of the total surviving interstitials. The effect of temperature on interstitial clustering in these adiabatic simulations is consistent with that observed by Gao, et al. ${ }^{12}$ in their work with a bybrid MD model that extracted heat from the simulation cell, i.e. the clustering fraction increases with temperature.

The interstitial cluster size distributions exhibit a somewhat consistent dependence on cascade energy and temperature as shown in Fig. 2, where the cascade energy dependence at $100 \mathrm{~K}$ is shown in Fig. 2(a). The influence of cascade temperature is shown for $20 \mathrm{keV}$ cascades in Fig, 2(b). All interstitial clusters larger than size 10 are combined into a single class in the histograms in Fig. 2. The interstitial cluster size distribution shifts to larger sizes as either the cascade energy or temperature increases. This can be seen as a decrease in mono-interstitials (size class 1), as well as an increase in the number of large clusters. At $100 \mathrm{~K}$, there are no interstitial clusters larger than 8 for cascade energies of $10 \mathrm{keV}$ or less. As discussed elsewhere, ${ }^{15}$ the effect of temperature appears to increase as the cascade energy increases. For example, the size distribution changes more between 100 and $600 \mathrm{~K}$ for $20 \mathrm{keV}$ cascades than it does between 100 and $900 \mathrm{~K}$ for $10 \mathrm{keV}$ cascades. The largest interstitial cluster observed contained 33 interstitials in a $20 \mathrm{keV}$ cascade at $600 \mathrm{~K}$.

\section{Vacancy Clustering}

The incascade vacancy clustering fraction is quite low ( $-3 \%$ of NRT) when the nearest neighbor criterion for clustering is applied. Because this observation was somewhat surprising, the spatial arrangement of the surviving vacancies was analyzed. The results of this analysis are shown in Fig. 3 for 10 to $50 \mathrm{keV}$ cascades at 100K. As mentioned, only a small number of first nearest neighbor $(1-n n)$ vacancy pairs are found. However, the peaks in the distribution at the second (2-nn) and fourth (4-nn) nearest neighbor locations are evidence of some spatial correlation of the vacancies. The peak at $2-\mathrm{nn}$ is consistent with the di-vacancy binding energy being greater for 2-nn $(0.22 \mathrm{eV})$ than for $1-\mathrm{nn}(0.09 \mathrm{eV}) ;{ }^{16}$ the reason for the peak at 4-nn is unclear. Since the time of the MD simulations is too short to allow vacancies to jump ( $\leq 100 \mathrm{ps}$ ), it seemed likely that these closely correlated vacancies might cluster over slightly longer times. This expectation has been confirmed in cascade annealing studies using Monte Carlo simulations. ${ }^{17}$ 

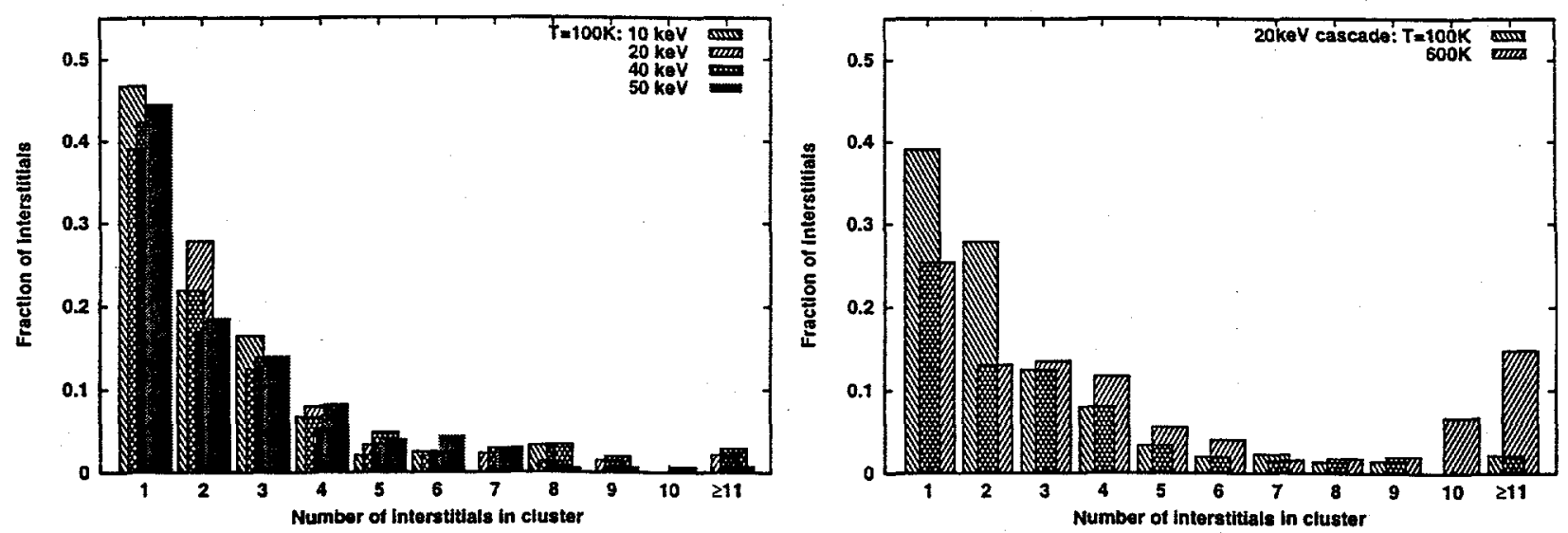

Fig. 2 Size distribution of interstitial clusters: (a) cascade energy dependence at $100 \mathrm{~K}$ and (b) 20 $\mathrm{keV}$ cascades at 100 and $600 \mathrm{~K}$. Size " 1 " denotes the mono-interstitial.

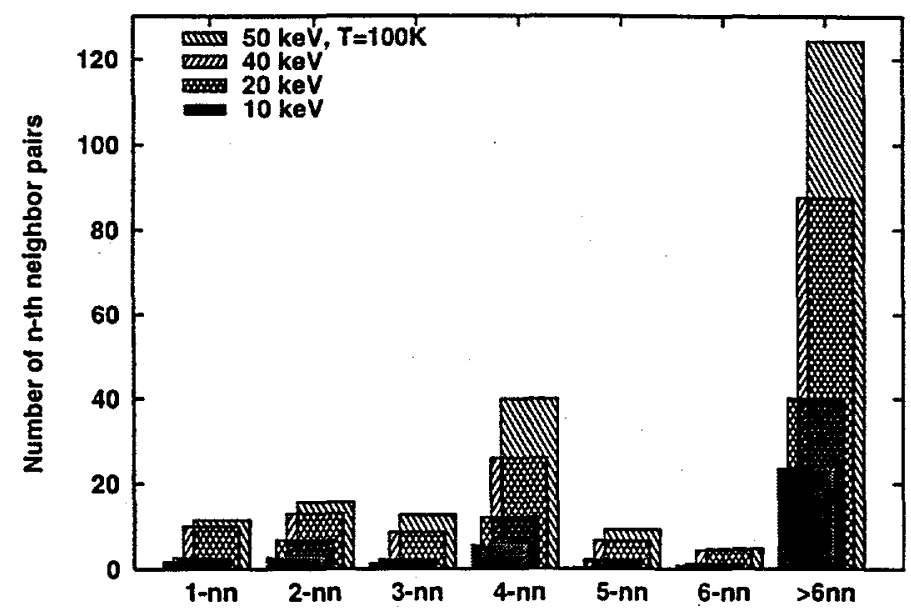

Fig. 3 Vacancy site correlation in 10 to 50 $\mathrm{keV}$ cascades at $100 \mathrm{~K}$, vacancies with another vacancy in first $(1-\mathrm{nn})$ to sixth (6-nn) nearest neighbor site.

Based on the results of the spatial correlation study and the cascade aging simulations, the vacancy clustering fraction was calculated with the clustering criterion relaxed to consider vacancies out to the fourth nearest neighbor. The in-cascade vacancy clustering fraction of the NRT displacements is shown in Fig. 4 for clustering criteria of 1-nn, 2-nn, 3-nn, and 4-nn. A comparison of Fig. 4 and Fig. 1 indicates that in-cascade vacancy clustering in iron remains lower

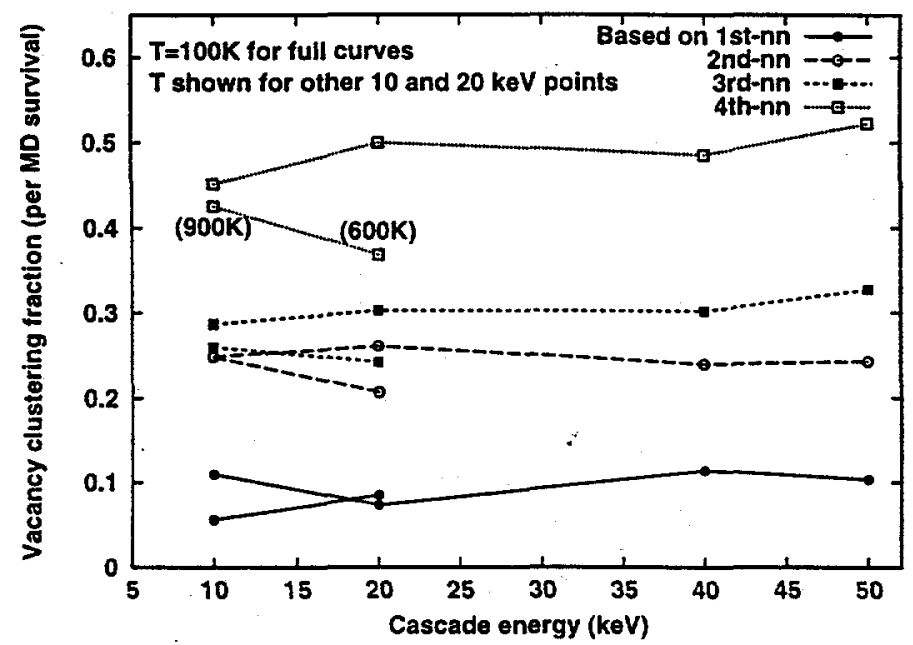

Fig. 4 Incascade vacancy clustering fraction for various clustering criteria: clustered vacancies divided by NRT displacements. 
than that of interstitials even when the largest nascent clusters are considered. This is consistent with the experimentally observed difficulty of forming vacancy clusters in iron as discussed in Ref. 5.

The effect of cascade energy and temperature on the vacancy cluster size distribution is shown in Fig. 5, for which the 4-nn clustering criterion has been used. Fig. 5 (a) illustrates that the vacancy cluster size distribution shifts to larger sizes as the cascade energy increases from 10 to $50 \mathrm{keV}$. This is similar to the change shown for interstitial clusters in Fig. 2 (a). There is a corresponding reduction in the fraction of single vacancies. However, the effect of cascade temperature shown in Fig. 5(b) is opposite to that observed for interstitials. The fraction of single vacancies increases and the size distribution shifts to smaller sizes as the temperature increases from 100 to $600 \mathrm{~K}$ for $20 \mathrm{keV}$ cascades.
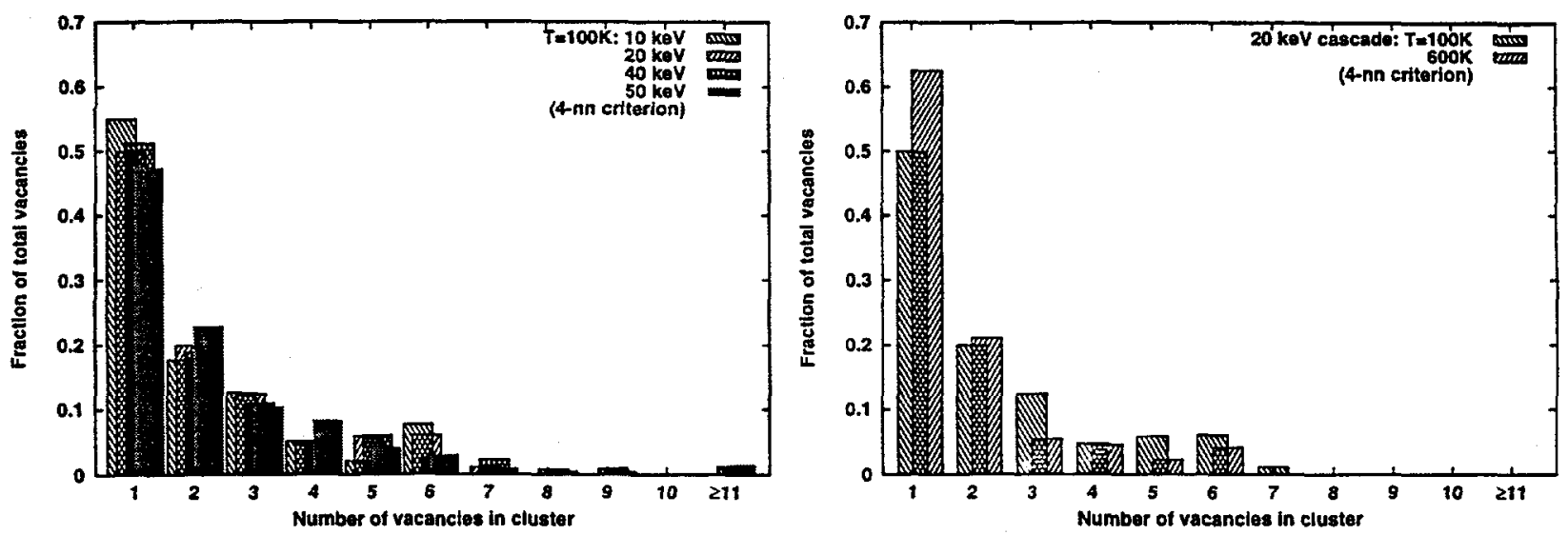

Fig. 5 Size distribution of vacancy clusters based on a 4-nn criterion: (a) cascade energy dependence at $100 \mathrm{~K}$ and (b) $20 \mathrm{keV}$ cascades at 100 and $600 \mathrm{~K}$. Size " 1 " denotes the mono-vacancy.

\section{SUMMARY}

As part of an ongoing effort to characterize primary damage formation in iron, $\mathrm{MD}$ displacement cascade simulations have been carried out at energies up to $50 \mathrm{keV}$ and temperatures up to $900 \mathrm{~K}$. A more detailed description of these simulations and a comparison with available experimental data will be published elsewhere. ${ }^{15}$ The results presented here have focused on in-cascade formation of vacancy and interstitial clusters as a significant component of that damage. Nearly $60 \%$ of the stable, surviving interstitials ( $20 \%$ of NRT) formed by high energy cascades are found in such clusters, with the largest cluster observed containing 33 interstitials. Much less in-cascade vacancy cluster formation is observed. Only about $10 \%$ of the stable vacancies ( $\sim 3 \%$ of NRT) are clustered if the criterion for cluster definition is vacancies in nearest neighbor locations. However, analysis of the spatial distribution of vacancies suggests that even the quenched-in cascade vacancies may be correlated out to the fourth nearest neighbor distance. Such a collection of vacancies can be considered a nascent vacancy cluster that is likely to coalesce when the vacancies have had time to make a few jumps.Accounting for vacancies out to the fourth nearest neighbor, the clustering fraction approaches $50 \%$ of the total or $15 \%$ of NRT.

The size distribution of both vacancy and interstitial clusters shifts to larger sizes as the cascade energy increases. The effect of temperature depends on the defect type. Increasing temperature leads to a further increase in number of large interstitial clusters, but a decrease in the number of large vacancy clusters. Because of low homogeneous nucleation rates for extended defect production, in-cascade clustering may play a significant role in defect nucleation in 
irradiated materials, thereby promoting microstructural evolution and concomitant mechanical property changes. In addition, the formation of larger clusters at higher cascade energies could lead to a greater effect of neutron or PKA energy spectrum than is predicted simply on the basis of total defect survival or the total clustering fraction. ${ }^{14}$

\section{ACKNOWLEGEMENTS}

$$
1886-N 695-3 W
$$

Research sponsored by the Office of Nuclear Regulatory Research, U.S. Nuclear Regulatory Commission under interagency agreement DOE 1-886-8109-8L with the U.S. Department of Energy, and by the Division of Materials Sciences and the Office of Fusion Energy Sciences, U.S. Department of Energy under contract DE-AC05-84OR21400 with Lockheed Martin Energy Research, Corp.

\section{REFERENCES}

1. R. S. Averback, T. Diaz de la Rubia, and R. Benedek, Nucl. Inst. and Meth. B33, 693 (1988).

2. A. J. E. Foreman, W. J. Phythian, and C. A. English, Phil. Mag. A66, 571 (1992).

3. A. F. Calder and D. J. Bacon, J. Nucl. Mater. 207, 25 (1993).

4. D. J. Bacon and T. Diaz de la Rubia, J. Nucl. Mater. 216, 275 (1994).

5. W. J. Phythian, R. E. Stoller, A. J. E. Foreman, A. F. Calder, and D. J. Bacon, J. Nucl. Mater. 223, 245 (1995).

6. R. E. Stoller, Microstructure of Irradiated Materials, Mat. Res. Soc. Symp. Proc. Vol. 373, Materials Research Society, Warrandale, PA, 1995, pp. 21-26.

7. D. J. Bacon, A. F. Calder, F. Gao, V. G. Kapinos, and S. J. Wooding, Nucl. Inst. and Meth. B102, 37 (1995).

8. R. E. Stoller, JOM (formerly Journal of Metals) 48, 23 (1996).

9. R. E. Stoller, G. R. Odette, and B. D. Wirth, J. Nucl. Mater. 251, 49 (1997).

10. M. W. Finnis, "MOLDY6-A Molecular Dynamics Program for Simulation of Pure Metals," AERE R-13182, UK AEA Harwell Laboratory (1988).

11. M. W. Finnis and J. E. Sinclair, Phil. Mag. A50, 45 (1984) and Erratum, Phil. Mag. A53, 161 (1986).

12. F. Gao, D. J. Bacon, P. E. J. Flewitt, and T. A. Lewis, J. Nucl. Mater. 249, 77 (1997).

13. M. J. Norgett, M. T. Robinson, and I. M. Torrens, Nucl. Eng. and Des. 33, 50 (1975).

14. R. E. Stoller and L. R. Greenwood, "From Molecular Dynamics to Kinetic Rate Theory: A Simple Example of Multiscale Modeling," these proceedings.

15. R. E. Stoller, "Primary Defect Formation in bcc Iron: The Role of Cascade Energy and Temperature and Pre-existing Defects," presented at the International Workshop: Basic Aspects of Differences in Irradiation Effects Between fcc, bcc, and hcp Metals and Alloys, Cangas de Onis, Spain, 15-20 October 1998, to be submitted to J. Nucl. Mater.

16. B. D. Wirth, "On the Character of Nano-scale Features in Reactor Pressure Vessel Steels Under Neutron Irradiation," Ph. D. Dissertation, University of California, Santa Barbara (1998).

17. B. D. Wirth and G. R. Odette, "Kinetic Lattice Monte Carlo Simulations of Cascade Aging in Iron and Dilute Iron-Copper Alloys," these proceedings. 